\title{
Some logarithmic Minkowski inequalities for nonsymmetric convex bodies and related problems
}

Lewen $\mathrm{Ji}^{1,2^{*}}$

\section{*Correspondence:}

jilewen2008@163.com

${ }^{1}$ Department of Mathematics, East

China University of Technology,

Nanchang, China

${ }^{2}$ Department of Mathematics,

Shanghai University, Shanghai, China

\section{Springer}

\begin{abstract}
In this paper, we show the existence of a solution to an even logarithmic Minkowski problem for $p$-capacity and prove some analogue inequalities of the logarithmic Minkowski inequality for general nonsymmetric convex bodies involving $p$-capacity.

MSC: $31 \mathrm{~B} 15 ; 52 \mathrm{~A} 20$

Keywords: Logarithmic Minkowski inequality; Logarithmic Minkowski problem; p-capacity
\end{abstract}

\section{Introduction}

A convex body in an $n$-dimensional Euclidean space $\mathbb{R}^{n}$ is a compact convex set that has nonempty interior. The cone-volume measure $V_{K}$ of a convex body $K$ is a Borel measure on the unit sphere $S^{n-1}$ defined for a Borel $\omega \in S^{n-1}$ by

$$
V_{K}(\omega)=\frac{1}{n} \int_{x \in g_{K}^{-1}(\omega)} x \cdot g_{K} d \mathcal{H}^{n-1}(x),
$$

where $g_{K}: \partial K \rightarrow S^{n-1}$ is the Gauss map of $K$, defined on $\partial K$, the set of boundary points of $K$ that have a unique outer unit normal, and $\mathcal{H}^{n-1}$ is an $(n-1)$-dimensional Hausdorff measure, see, e.g., $[5,20,24,28]$. The cone-volume measure of a convex body has clear geometric significance. Böröczky et al. in [4] posed the subspace concentration condition and completely solved the even Minkowski problem. The problem asks: What are the necessary and sufficient conditions on a finite Borel measure $\mu$ on $S^{n-1}$ such that $\mu$ is the conevolume measure of a convex body in $\mathbb{R}^{n}$ ? In [30], Zhu solved the case of discrete measures whose supports are in general position. Uniqueness for the logarithmic Minkowski problem was completely settled for even measures in $\mathbb{R}^{2}$ in [3]. Recently, Stancu [23] proved the logarithmic Minkowski inequality for nonsymmetric convex bodies. Wang, $\mathrm{Xu}$, and Zhou [25] gave the $L_{p}$ version of Stancu's results. For more results, see, e.g., [2, 4, 21, 25, 26, 30].

In his celebrated paper [17], Jerison solved the Minkowski problem for the capacitary measure, the measure that is the variational functional arising from the electrostatic capacity. Colesanti et al. in [9] extended Jerison's work on electrostatic capacity to $p$-capacity.

(c) The Author(s) 2020. This article is licensed under a Creative Commons Attribution 4.0 International License, which permits use, sharing, adaptation, distribution and reproduction in any medium or format, as long as you give appropriate credit to the original author(s) and the source, provide a link to the Creative Commons licence, and indicate if changes were made. The images or other third party material in this article are included in the article's Creative Commons licence, unless indicated otherwise in a credit line to the material. If material is not included in the article's Creative Commons licence and your intended use is not permitted by statutory regulation or exceeds the permitted use, you will need to obtain permission directly from the copyright holder. To view a copy of this licence, visit http://creativecommons.org/licenses/by/4.0/. 
Naturally, the Minkowski problem for $p$-capacity was posed [9]: Given a finite Borel measure $\mu$ on $S^{n-1}$, what are the necessary and sufficient conditions on $\mu$ so that $\mu$ is the $p$ capacitary measure $\mu_{p}(K, \cdot)$ of convex body $K$ in $\mathbb{R}^{n}$ ? The authors in [9] proved the uniqueness of the solution when $1<p<n$ and existence and regularity when $1<p<2$, the existence for $2<p<n$ was solved by Akman et al. [1]. Inspired by the $L_{p}$ Minkowski problem for volume, Zou and Xiong [31] initiated the research into the $L_{q}$ Minkowski problem for $p$-capacitary measure. For more results, see, e.g., [6-8, 15-17, 19, 27, 29].

\subsection{Main results}

In this paper, we study the even logarithmic Minkowski problem and logarithmic Minkowski inequality for $p$-capacity. Our first result is to solve the existence part of the even logarithmic Minkowski problem for $p$-capacity. The problem asks: What are the necessary and sufficient conditions on a finite Borel measure $\mu$ on $S^{n-1}$ such that $\mu$ is the $L_{0} p$ capacitary measure of an origin-symmetric convex body in $\mathbb{R}^{n}$ ? Our proof is based on the techniques in $[4,9,23]$. In order to solve the existence of the even logarithmic Minkowski problem, we use the definition of subspace concentration inequality in [4].

Definition 1.1 ([4]) A finite Borel measure $\mu$ on $S^{n-1}$ is said to satisfy the subspace concentration inequality if, for every subspace $\xi$ of $\mathbb{R}^{n}$ such that $0<\operatorname{dim} \xi<n$,

$$
\mu\left(\xi \cap S^{n-1}\right) \leq \frac{1}{n} \mu\left(S^{n-1}\right) \operatorname{dim} \xi
$$

The measure is said to satisfy the subspace concentration condition if, in addition to satisfying the subspace concentration inequality (1.1), whenever

$$
\mu\left(\xi \cap S^{n-1}\right)=\frac{1}{n} \mu\left(S^{n-1}\right) \operatorname{dim} \xi
$$

for some subspace $\xi$, then there exists a subspace $\xi^{\prime}$, which is complementary to $\xi$ in $\mathbb{R}^{n}$, so that

$$
\mu\left(\xi^{\prime} \cap S^{n-1}\right)=\frac{1}{n} \mu\left(S^{n-1}\right) \operatorname{dim} \xi^{\prime}
$$

Theorem 1.1 Let $\mu$ be a nonzero finite even Borel measure on $S^{n-1}$ and $1<p<2$. Suppose that $\mu$ satisfies the strict subspace concentration inequality and $\mu(\{-u\})=0$ whenever $\mu(\{u\})>0$ for $u \in S^{n-1}$. Then the measure $\mu$ is the $L_{0} p$-capacitary measure of an originsymmetric convex body in $\mathbb{R}^{n}$.

Next, we prove the modified logarithmic Minkowski inequality for $p$-capacity.

Theorem 1.2 Suppose $K, L \in \mathcal{K}_{o}^{n}$ and $1<p<n$. Then

$$
\int_{S^{n-1}} \ln \left(\frac{h_{K}(u)}{h_{L}(u)}\right) d C_{p}^{*}(L, K, u) \geq \ln \left(\frac{C_{p}(L, K)}{C_{p}(L)}\right) \geq \frac{1}{n-p} \ln \left(\frac{C_{p}(K)}{C_{p}(L)}\right) .
$$

Equality holds if and only if $K$ and $L$ are homothetic. 


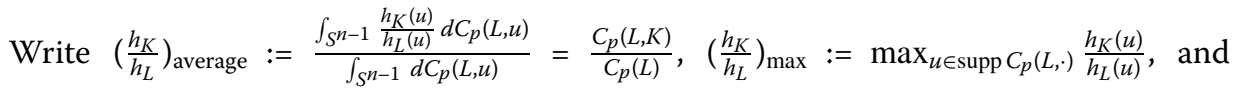

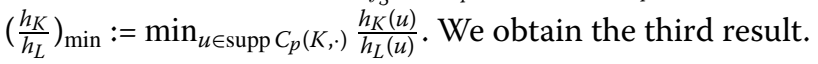

Theorem 1.3 Suppose $K, L \in \mathcal{K}_{o}^{n}$ with $L \subseteq K$ and $1<p<n$, then

$$
\int_{S^{n-1}} \ln \left(\frac{h_{K}(u)}{h_{L}(u)}\right) d C_{p}^{*}(L, u) \geq \frac{\left(\frac{h_{K}}{h_{L}}\right)_{\text {average }}}{\left(\frac{h_{K}(u)}{h_{L}(u)}\right)_{\max }} \frac{1}{n-p} \ln \left(\frac{C_{p}(K)}{C_{p}(L)}\right) .
$$

Equality holds if and only if $K=L$.

In general, we have

$$
\begin{aligned}
\int_{S^{n-1}} \ln \left(\frac{h_{K}(u)}{h_{L}(u)}\right) d C_{p}^{*}(L, u) \geq & \frac{\left(\frac{h_{K}}{h_{L}}\right)_{\text {average }}}{\left(\frac{h_{K}(u)}{h_{L}(u)}\right)_{\max }} \frac{1}{n-p} \ln \left(\frac{C_{p}(K)}{C_{p}(L)}\right) \\
& +\ln \left(\left(\frac{h_{K}}{h_{L}}\right)_{\min }\right)\left(1-\frac{\left(\frac{h_{K}}{h_{L}}\right)_{\text {average }}}{\left(\frac{h_{K}}{h_{L}}\right)_{\max }}\right) .
\end{aligned}
$$

This paper is organized as follows. In Sect. 2, we give a minimization problem for $p$ capacity. In Sect. 3, we give the proof of Theorem 1.1. In Sect. 4, we prove Theorem 1.2 and its application.

\subsection{Preliminaries}

For quick reference, we collect some basic facts on the theory of convex bodies. Good references are the books by Schneider [22].

Denote by $\mathcal{K}^{n}$ the set of convex bodes in $\mathbb{R}^{n}$ and by $\mathcal{K}_{o}^{n}$ the set of convex bodies with the origin $o$ in its interiors. Let $h_{K}$ and $h_{L}$ be the support functions $\left(h_{K}(u)=h(K, u):=\right.$ $\max _{u \in S^{n-1}}\{x \cdot u: x \in K\}$, where $x \cdot u$ denotes the inner product of $u$ and $x$ ) of $K$. Let $I \subset$ $\mathbb{R}$ be an interval containing $0, C^{+}\left(S^{n-1}\right)$ be a class of continuous and positive functions on $S^{n-1}, C_{e}^{+}\left(S^{n-1}\right)$ means the subsets of $C^{+}\left(S^{n-1}\right)$ are the even functions, and assume that $h_{t}(u)=h(t, u): I \times S^{n-1} \rightarrow(0,+\infty)$ is continuous. Put

$$
\Omega_{t}=\bigcap_{u \in S^{n-1}}\left\{x \in \mathbb{R}^{n}: x \cdot u \leq h_{t}(u)\right\} .
$$

The convex body $\Omega_{t}$ is called the Aleksandrov body associated with $h_{t}$. Via the support function, Böröczky et al. in [3] defined the log Minkowski combination $(1-\lambda) \cdot K+_{0} \lambda \cdot L$, that is,

$$
(1-\lambda) \cdot K+_{0} \lambda \cdot L=\bigcap_{u \in S^{n-1}}\left\{x \in \mathbb{R}^{n}: x \cdot u \leq h_{K}(u)^{1-\lambda} h_{L}(u)^{\lambda}\right\} .
$$

The surface area measure $S_{K}$ of a convex body $K$ is a Borel measure on the unit sphere $S^{n-1}$ defined for a Borel $\omega \in S^{n-1}$ by

$$
S_{K}(\omega)=\int_{x \in g_{K}^{-1}(\omega)} d \mathcal{H}^{n-1}(x)
$$


Let $\Omega$ be a bounded convex domain in $\mathbb{R}^{n}, n \geq 3, \bar{\Omega}$ be its closure. The equilibrium potential $u=u_{\Omega}$ is the unique solution to the boundary value problem

$$
\left\{\begin{array}{l}
\Delta_{p} u=0 \quad \text { in } \mathbb{R}^{n} \backslash \bar{\Omega}, \\
u=1 \quad \text { on } \partial \Omega \\
\lim _{|x| \rightarrow \infty} u(x)=0
\end{array}\right.
$$

where $\Delta_{p}$ is the $p$-Laplace operator and $1<p<n$. Due to Dahlbeg [12], we can see that $\nabla u$ has non-tangential limits almost everywhere on $\partial \Omega$ and $|\nabla u| \in L^{p}\left(\partial \Omega, \mathcal{H}^{n-1}\right)$. The $p$-capacity was defined by

$$
C_{p}(\Omega)=\inf \left\{\int_{\mathbb{R}^{n}}\left|\nabla u_{\Omega}\right|^{p} d x: u \in C_{c}^{\infty}\left(\mathbb{R}^{n}\right), u \geq 1 \text { on } \Omega\right\}
$$

where $C_{c}^{\infty}\left(\mathbb{R}^{n}\right)$ is the set of $C^{\infty}$ functions in $\mathbb{R}^{n}$ with compact support. In [9], the authors proved the following Hadamard variational formula for $p$-capacity. Let $K, L \in \mathcal{K}_{o}^{n}$ and $1<$ $p<n$,

$$
\left.\frac{d}{d t} C_{p}(K+t L)\right|_{t=0^{+}}=(p-1) \int_{S^{n-1}} h(L, u) d \mu_{p}(K, u),
$$

where the $p$-capacitary measure $\mu_{p}(K, E)$ is defined by

$$
\mu_{p}(K, E)=\int_{g_{K}^{-1}(E)}\left|\nabla u_{K}\right|^{p} d \mathcal{H}^{n-1}
$$

and the Poincaré $p$-capacity formula

$$
C_{p}(K)=\frac{p-1}{n-p} \int_{S^{n-1}} h(K, u) d \mu_{p}(K, u)=\frac{p-1}{n-p} \int_{\partial K}|\nabla u|^{p}\left(x \cdot g_{K}\right) d \mathcal{H}^{n-1}(x) \text {. }
$$

\section{A minimization problem}

In this section, we study a minimization problem, its solution also solves the logarithmic Minkowski problem for $p$-capacity. The following lemma will be needed.

Lemma 2.1 ([9]) Let $h(t, u): I \times S^{n-1} \rightarrow(0,+\infty)$ be continuous and $\left\{K_{t}\right\}_{t \in I}$ be the family of Aleksandrov domain associated with $h_{t}$. If

$$
h_{+}^{\prime}(0, u)=\lim _{t \rightarrow 0^{+}} \frac{h(t, u)-h(0, u)}{t}
$$

is uniform on $S^{n-1}$, then

$$
\lim _{t \rightarrow 0^{+}} \frac{C_{p}\left(K_{t}\right)-C_{p}\left(K_{0}\right)}{t}=(p-1) \int_{S^{n-1}} h_{+}^{\prime}(0, u) d \mu_{p}\left(K_{0}, u\right) .
$$

With Lemma 2.1 in hand, we use the definition of log Minkowski combination to prove the following result. 
Lemma 2.2 Let $K \in \mathcal{K}_{o}^{n}$ and $f \in C^{+}\left(S^{n-1}\right)$ be nonnegative. If $1<p<n$, then

$$
\lim _{t \rightarrow 0^{+}} \frac{C_{p}\left(K+_{0} t \cdot f\right)-C_{p}(K)}{t}=(p-1) \int_{S^{n-1}} h(K, u) \ln f d \mu_{p}(K, u) .
$$

Proof Let $0<t_{0}<+\infty$, consider the interval $\left[0, t_{0}\right]$. We have

$$
h_{+}^{\prime}(0, u)=\lim _{t \rightarrow 0^{+}} \frac{h(t, u)-h(0, u)}{t}=h(K, u) \ln f \quad \text { for all } u \in S^{n-1}
$$

Then, by Lemma 2.1, we obtain

$$
\lim _{t \rightarrow 0^{+}} \frac{C_{p}\left(K+_{0} t \cdot f\right)-C_{p}(K)}{t}=(p-1) \int_{S^{n-1}} h(K, u) \ln f d \mu_{p}(K, u) .
$$

Corollary 2.1 Suppose $K, L \in \mathcal{K}_{o}^{n}$ and $1<p<n$, then

$$
\lim _{t \rightarrow 0^{+}} \frac{C_{p}\left(K+_{0} t \cdot L\right)-C_{p}(K)}{t}=(p-1) \int_{S^{n-1}} h(K, u) \ln h(L, u) d \mu_{p}(K, u) .
$$

In view of the above result, we give the following definitions.

Definition 2.1 Let $1<p<n$ and $K \in \mathcal{K}_{o}^{n}$, then the Borel measure $\mu_{p, 0}(K, \cdot)$ on $S^{n-1}$, defined by

$$
\mu_{p, 0}(K, \omega)=\int_{\omega} h(K, u) d \mu_{p}(K, u)
$$

for $\omega \subset S^{n-1}$, is called the $L_{0} p$-capacitary measure of $K$.

Definition 2.2 Let $1<p<n$ and $K, L \in \mathcal{K}_{o}^{n}$, then $L_{0}$ mixed $p$-capacity of $K$ and $L$ is defined by

$$
C_{p, 0}(K, L)=\frac{p-1}{n-p} \int_{S^{n-1}} h(K, u) \ln h(L, u) d \mu_{p}(K, u)
$$

The next lemma shows that $C_{p, 0}(K, L)$ is continuous in $(K, L)$.

Lemma 2.3 Let $K_{i}, L_{i}, K, L \in \mathcal{K}_{o}^{n}$ and $1<p<n$. Assume that $\left(K_{i}, L_{i}\right) \rightarrow(K, L)$ as $i \rightarrow \infty$, then $C_{p, 0}\left(K_{i}, L_{i}\right) \rightarrow C_{p, 0}(K, L)$.

Proof By the assumption $\left(K_{i}, L_{i}\right) \rightarrow(K, L)$, we have $h_{K_{i}} \rightarrow h_{K}, h_{L_{i}} \rightarrow h_{L}$ uniformly on $S^{n-1}$, and $\mu_{p}\left(K_{i}, \cdot\right) \rightarrow \mu_{p}(K, \cdot)$ weakly. It follows that $h_{K_{i}} \ln h_{L_{i}} \rightarrow h_{K} \ln h_{L}$ uniformly on $S^{n-1}$. By Definition 2.2, the desired limit is obtained.

The weak convergence of $p$-capacitary measure implies the weak convergence of $\mu_{p, 0}$ as follows.

Lemma 2.4 Let $K_{i}, K \in \mathcal{K}_{o}^{n}$ and $1<p<n$. If $K_{i} \rightarrow K$ as $i \rightarrow \infty$, then $\mu_{p, 0}\left(K_{i}\right) \rightarrow \mu_{p, 0}(K)$ weakly. 
We now consider the minimization problem

$$
\inf \left\{\Phi_{\mu}(K): C_{p}(K)=|\mu| \text { and } K \in \mathcal{K}_{e}^{n}\right\}
$$

where $\mathcal{K}_{e}^{n}$ is an origin-symmetric convex body with nonempty interior, $\mu$ is a finite even Borel measure on $S^{n-1}$ with total mass $|\mu|>0$, and the logarithmic functional $\Phi_{\mu}: \mathcal{K}_{e}^{n} \rightarrow \mathbb{R}$ is defined by

$$
\Phi_{\mu}(K)=\int_{S^{n-1}} \ln h_{K} d \mu .
$$

The following theorem shows a solution to the logarithmic Minkowski type problem for the measure $\mu$ is a solution to minimization problem for the function $\Phi_{\mu}$.

Theorem 2.1 Let $\mu$ be a finite even Borel measure on $S^{n-1}$ with $|\mu|>0$ and $1<p<n$. If $K$ is an origin-symmetric convex body such that $C_{p}(K)=|\mu|$ and

$$
\Phi_{\mu}(K)=\inf \left\{\Phi_{\mu}(Q): C_{p}(Q)=|\mu|, Q \in \mathcal{K}_{e}^{n}\right\}
$$

then the measure $\mu$ is the $L_{0} p$-capacitary measure of $K$.

Proof Clearly, we may assume that $\mu$ is a probability measure. Next, we consider the minimization problem

$$
\inf _{f \in \mathcal{C}_{e}^{+}\left(S^{n-1}\right)} F(f)
$$

where the continuous functional $F: \mathcal{C}_{e}^{+}\left(S^{n-1}\right) \rightarrow(0,+\infty)$ by

$$
F(f)=\frac{\exp \int_{S^{n-1}} \ln f d \mu}{C_{p}\left(K_{f}\right)^{\frac{1}{n-p}}},
$$

here $K_{f}$ denotes the Wulff shape of $f \in \mathcal{C}_{e}^{+}\left(S^{n-1}\right)$. Notice that $F$ is homogeneous of degree 0, i.e., $F(s f)=F(f)$ for $s>0$. By the properties of Aleksandrov body, we have $h_{K_{f}} \leq f$. According to Lemma 2.1 in [31], we have $C_{p}(f)=C_{p}\left(K_{f}\right)$; in addition, $C_{p}\left(K_{f}\right)=C_{p}\left(h_{K_{f}}\right)$, which yields $F\left(K_{f}\right) \leq F(f)$. Therefore, we shall search for the infimum of $F$ among the support functions of origin-symmetric convex bodies. It follows that the infimum of $F$

$$
\inf _{f \in \mathcal{C}_{e}^{+}\left(S^{n-1}\right)} F(f)=\inf \left\{\exp \left(\Phi_{\mu}(Q)\right): C_{p}(Q)=1, Q \in \mathcal{K}_{e}^{n}\right\}
$$

Obviously, the right infimum is attained at $K \in \mathcal{K}_{e}^{n}$. Thus, the support function $h_{K}>0$ is a solution of minimization problem, i.e.,

$$
\inf _{f \in \mathcal{C}_{\mathcal{e}}^{+}\left(S^{n-1}\right)} F(f)=F\left(h_{K}\right)
$$

Given the function $h_{t}=h(\cdot, t): S^{n-1} \times \mathbb{R} \rightarrow(0, \infty)$ is defined by

$$
h_{t}=h(\cdot, t)=h_{K} \exp (t f),
$$


by the function $F\left(h_{t}\right)$ has a minimum at $t=0$, this implies that

$$
\frac{d}{d t} F\left(h_{t}\right)=0
$$

On the other hand,

$$
\lim _{t \rightarrow 0} \frac{h_{t}-h_{0}}{t}=f h_{K}, \quad \text { uniformly on } S^{n-1},
$$

it follows from $C_{p}(K)=1$ that

$$
\left[-\frac{p-1}{n-p} \int_{S^{n-1}} f(u) h_{K}(u) d \mu_{p}(K, u)+\int_{S^{n-1}} f(u) d \mu(u)\right] \exp \left(\int_{S^{n-1}} \ln h_{K}(u) d \mu(u)\right)=0
$$

which yields

$$
\frac{p-1}{n-p} \int_{S^{n-1}} f(u) d \mu_{p, 0}(K, u)=\int_{S^{n-1}} f(u) d \mu(u) .
$$

Since $f \in \mathcal{C}_{e}^{+}\left(S^{n-1}\right)$ is arbitrary, we conclude that

$$
d \mu(\cdot)=\frac{p-1}{n-p} d \mu_{p, 0}(K, \cdot)
$$

this completes the proof of the theorem.

\section{Logarithmic Minkowski problem}

In the previous section, we have proved the existence of a solution to the logarithmic Minkowski problem by using the variational argument. In this section, we show the proof of the main result.

Lemma 3.1 Let $\mu$ be an even finite Borel measure on $S^{n-1}$ satisfying the strict subspace concentration inequality. If $1<p<n$ and $\mu(\{-u\})=0$ whenever $\mu(\{u\})>0$, then there exists an origin-symmetric convex body $K$ so that

$$
\inf \left\{\Phi_{\mu}(Q): C_{p}(Q)=|\mu|, Q \in \mathcal{K}_{e}^{n}\right\}=\int_{S^{n-1}} \ln h_{K} d \mu
$$

Proof Without loss of generality, we assume that $|\mu|=1$. Let $Q_{l}$ be the minimizing sequence of origin-symmetric convex bodies, that is, $Q_{l}$ satisfies the $C_{p}\left(Q_{l}\right)=1$ and

$$
\lim _{l \rightarrow \infty} \Phi_{\mu}\left(Q_{l}\right)=\inf _{Q \in \mathcal{K}_{e}^{n}}\left\{\Phi_{\mu}(Q): C_{p}(Q)=1, Q \in \mathcal{K}_{e}^{n}\right\}
$$

Taking $L=\gamma^{-\frac{1}{n-p}} B_{n}, \gamma=\left(\frac{p-1}{n-p}\right)^{1-p} \omega_{n}$, here $B_{n}$ is a unit ball. Then $C_{p}(L)=1$, it follows that

$$
\lim _{l \rightarrow \infty} \Phi_{\mu}\left(Q_{l}\right) \leq-\frac{1}{n-p} \ln \gamma
$$


By John's theorem [18] associated with $Q_{l}$, there exists an origin-symmetric ellipsoid $E_{l}$ so that

$$
E_{l} \subseteq Q_{l} \subseteq \sqrt{n} E_{l} .
$$

Note that, for every origin-symmetric ellipsoid $E_{l}$, there is a cross-polytope $P_{l}$ denoted by $P_{l}=\left[ \pm a_{1 l} u_{1 l}, \ldots, \pm a_{n l} u_{n l}\right]$ such that $P_{l} \subseteq E_{l} \subseteq \sqrt{n} P_{l}$. Hence, $P_{l} \subseteq Q_{l} \subseteq n P_{l}$. It follows from $C_{p}\left(Q_{l}\right)=1$ that $C_{p}\left(P_{l}\right) \geq n^{-\frac{1}{n-p}}$. We next claim that suppose $K$ is a convex body containing the origin and satisfying $V(K)=0$, then $C_{p}(K)=0$. In fact, since

$$
\begin{aligned}
0 & =V(K)=\frac{1}{n} \int_{S^{n-1}} h_{K}(u) d S(K, u) \\
& =\frac{1}{n} \int_{\left\{h_{K}>0\right\}} h_{K}(u) d S(K, u)+\frac{1}{n} \int_{\left\{h_{K}=0\right\}} h_{K}(u) d S(K, u) \\
& =\frac{1}{n} \int_{\left\{h_{K}>0\right\}} h_{K}(u) d S(K, u) .
\end{aligned}
$$

It follows that $S\left(K,\left\{h_{K}>0\right\}\right)=0$, which implies $\mathcal{H}^{n-1}\left(g_{K}^{-1}\left(\left\{h_{K}>0\right\}\right)\right)=0$. Together with the fact that $|\nabla u|^{p}$ is integrable on $\partial K$, we have

$$
\mu_{p}\left(K,\left\{h_{K}>0\right\}\right)=\int_{g_{K}^{-1}\left(\left\{h_{K}>0\right\}\right)}|\nabla u|^{p} \mathcal{H}^{n-1}(x)=0 .
$$

By (1.5), we have $C_{p}(K)=0$. Thus, there exists a constant $c>0$ such that $V(K) \geq c C_{p}(K)$. This yields

$$
\prod_{i=1}^{n} a_{i l}=\frac{n ! V\left(P_{l}\right)}{2^{n}} \geq \frac{c n ! C_{p}\left(P_{l}\right)}{2^{n}}:=\gamma_{1}
$$

Assume that $Q_{l}$ is not bounded. Then $P_{l}$ is not bounded, thus there exists $a_{n l}$ such that $\lim _{l \rightarrow \infty} a_{n l}=\infty$. Applying Lemma 6.2 in [3] to $P_{l}^{\prime}=\gamma_{1}^{-\frac{1}{n}} P_{l}$ implies that $\Phi_{\mu}\left(P_{l}^{\prime}\right)$ is not bounded, which gives that $\Phi_{\mu}\left(Q_{l}\right)$ is not bounded. This contradicts (3.1). Therefore, the sequence $Q_{l}$ is bounded. By Blaschke's selection theorem, $Q_{l}$ has a subsequence that converges to an origin-symmetric convex body $K$. In the following, we prove $K \in \mathcal{K}_{o}^{n}$ is $n$ dimensional. Let $\operatorname{dim} K \leq n-2$. Since $1<p<2$, we have $\operatorname{dim} K \leq n-2<n-p$, so $C_{p}(K)=0$ by [13], p. 179, which contradicts that $C_{p}(K)=1$. Let $\operatorname{dim} K=n-1$, there exists a unit vector $u \in S^{n-1}$ such that $K \subset u^{\perp}$. Then $u,-u \in \operatorname{supp} \mu$. But $\mu$ satisfies $\mu(\{-u\})=0$ whenever $\mu(\{u\})>0$ for any $u \in S^{n-1}$, which is a contradiction. This gives the desired result.

We get the proof of Theorem 1.1 directly from Theorem 2.1 and Lemma 3.1.

\section{Logarithmic Minkowski type inequality}

In this section, we set $d C_{p}(L, K, \cdot)=\frac{1}{n-p} h_{K} d \mu_{p}(L, \cdot)$ and $d C_{p}(L, \cdot)=\frac{1}{n-p} h_{L} d \mu_{p}(L, \cdot)$. Then $C_{p}(L, K)=\int_{S^{n-1}} d C_{p}(L, K, u)$ and $C_{p}(L)=\int_{S^{n-1}} d C_{p}(L, u)$. Clearly,

$$
d C_{p}^{*}(L, K, \cdot)=\frac{1}{C_{p}(L, K)} d C_{p}(L, K, \cdot)
$$


and $d C_{p}^{*}(L, \cdot)=\frac{1}{C_{p}(L)} d C_{p}(L, \cdot)$ are their normalization, respectively. Now, we give the proof of Theorem 1.2.

Proof of Theorem 1.2 Consider the function $f:[1,+\infty] \rightarrow \mathbb{R}$ defined by

$$
f(q)=\frac{1}{C_{p}(L, K)} \int_{S^{n-1}}\left(\frac{h_{K}}{h_{L}}\right)^{\frac{q}{q+n}} d C_{p}(L, u) .
$$

According to Lebesgue's dominated convergence theorem, we obtain: as $q \rightarrow \infty$,

$$
\int_{S^{n-1}}\left(\frac{h_{K}(u)}{h_{L}(u)}\right)^{\frac{q}{q+n}} d C_{p}(L, u) \rightarrow C_{p}(L, K)
$$

and

$$
\int_{S^{n-1}}\left(\frac{h_{K}(u)}{h_{L}(u)}\right)^{\frac{q}{q+n}} \ln \left(\frac{h_{K}(u)}{h_{L}(u)}\right) d C_{p}(L, u) \rightarrow \int_{S^{n-1}} \frac{h_{K}(u)}{h_{L}(u)} \ln \left(\frac{h_{K}(u)}{h_{L}(u)}\right) d C_{p}(L, u) .
$$

Using L'Hôpital's rule, we have

$$
\begin{aligned}
\lim _{q \rightarrow \infty} \ln (f(q))^{q+n} & =\lim _{q \rightarrow \infty} \frac{\frac{n}{(n+q)^{2} C_{p}(L, K)} \int_{S^{n-1}}\left(\frac{h_{K}(u)}{h_{L}(u)}\right)^{\frac{q}{q+n}} \ln \left(\frac{h_{K}(u)}{h_{L}(u)}\right) d C_{p}(L, u)}{-\frac{f(q)}{(q+n)^{2}}} \\
& =-\frac{n}{C_{p}(L, K)} \int_{S^{n-1}} \frac{h_{K}(u)}{h_{L}(u)} \ln \left(\frac{h_{K}(u)}{h_{L}(u)}\right) d C_{p}(L, u) \\
& =-\frac{n}{C_{p}(L, K)} \int_{S^{n-1}} \ln \left(\frac{h_{K}(u)}{h_{L}(u)}\right) d C_{p}(L, K, u) .
\end{aligned}
$$

By (4.1) and (4.2), we have

$$
\begin{aligned}
& \exp \left[-n \int_{S^{n-1}} \ln \left(\frac{h_{K}(u)}{h_{L}(u)}\right) d C_{p}^{*}(L, K, u)\right] \\
& \quad=\lim _{q \rightarrow \infty}\left[\frac{1}{C_{p}(L, K)} \int_{S^{n-1}}\left(\frac{h_{K}(u)}{h_{L}(u)}\right)^{\frac{q}{q+n}} d C_{p}(L, u)\right]^{n+q} .
\end{aligned}
$$

The reverse Hölder inequality gives

$$
\left(\int_{S^{n-1}}\left(\frac{h_{K}(u)}{h_{L}(u)}\right)^{\frac{q}{q+n}} d C_{p}(L, u)\right)^{\frac{n+q}{q}}\left(\int_{S^{n-1}} d C_{p}(L, u)\right)^{-\frac{n}{q}} \leq C_{p}(L, K) .
$$

Equality holds if and only if $K$ is homothetic to $L$. This gives the first inequality of Theorem 1.2.

Now, we prove the second inequality of Theorem 1.2. Using Minkowski's inequality for $p$-capacity [10], we have

$$
C_{p}(L, K) \geq C_{p}(K)^{\frac{1}{n-p}} C_{p}(L)^{1-\frac{1}{n-p}} .
$$

Equality holds if and only if $K$ is homothetic to $L$. 
Similarly, we obtain the reverse form of Theorem 1.2.

Theorem 4.1 Let $K, L \in \mathcal{K}_{o}^{n}$ and $1<p<n$. Then

$$
\int_{S^{n-1}} \ln \frac{h_{K}(u)}{h_{L}(u)} d C_{p}^{*}(L, u) \leq \ln \frac{C_{p}(L, K)}{C_{p}(L)} \leq \int_{S^{n-1}} \ln \left(\frac{h_{L}(u)}{h_{K}(u)}\right) d C_{p}^{*}(L, K, u),
$$

with equality if and only if $K$ is homothetic to $L$.

First proof Via the same idea in Theorem 1.2, we have

$$
\begin{aligned}
& \exp \left(-\frac{1}{C_{p}(L)} \int_{S^{n-1}} \ln \left(\frac{h_{K}(u)}{h_{L}(u)}\right) d C_{p}(L, u)\right) \\
& \quad=\lim _{q \rightarrow \infty}\left[\frac{1}{C_{p}(L)} \int_{S^{n-1}}\left(\frac{h_{K}(u)}{h_{L}(u)}\right)^{\frac{1}{q+n}} d C_{p}(L, u)\right]^{n+q} .
\end{aligned}
$$

From the reverse Hölder inequality, we have

$$
\left(\int_{S^{n-1}}\left(\frac{h_{K}(u)}{h_{L}(u)}\right)^{\frac{1}{q+n}} d C_{p}(L, u)\right)^{q+n}\left(\int_{S^{n-1}} d C_{p}(L, u)\right)^{1-q-n} \leq C_{p}(L, K) .
$$

Thus,

$$
\frac{1}{C_{p}(L)} \int_{S^{n-1}} \ln \left(\frac{h_{K}}{h_{L}}\right) d C_{p}(L) \leq \ln \left(\frac{C_{p}(L, K)}{C_{p}(L)}\right) .
$$

Second proof We will use Gibbs' inequality [11], i.e., let $f$ and $g$ be the probability density functions on a measure space $(X, v)$, then $\int f \ln f d v \geq \int f \ln g d v$, equality holds if and only if $f=g$. On the one hand, let $f d v(\cdot)=\frac{h_{L}}{h_{K} C_{p}(L)} d C_{p}(L, K, \cdot)$ and $g d v(\cdot)=\frac{1}{C_{p}(L, K)} d C_{p}(L, K, \cdot)$. By Gibbs' inequality in [11], we have

$$
\begin{gathered}
\int_{S^{n-1}} \frac{h_{L}(u)}{h_{K}(u) C_{p}(L)} \ln \left(\frac{h_{L}(u)}{h_{K}(u) C_{p}(L)}\right) d C_{p}(L, K, u) \\
\geq \int_{S^{n-1}} \frac{1}{C_{p}(L, K)} \ln \left(\frac{1}{C_{p}(L, K)}\right) d C_{p}(L, K, u),
\end{gathered}
$$

which implies that

$$
\int_{S^{n-1}} \ln \left(\frac{h_{L}(u)}{h_{K}(u)}\right) d C_{p}^{*}(L, u) \leq \ln \left(\frac{C_{p}(L, K)}{C_{p}(L)}\right)
$$

According to the equality condition, we know that $K$ is homothetic to $L$.

On the other hand, we set $f d v(\cdot)=\frac{1}{C_{p}(L, K)} d C_{p}(L, K, \cdot)$ and $g d v(\cdot)=\frac{h_{L}}{h_{K} C_{p}(L)} d C_{p}(L, K, \cdot)$, then

$$
\begin{aligned}
& \int_{S^{n-1}} \frac{1}{C_{p}(L, K)} \ln \left(\frac{1}{C_{p}(L, K)}\right) d C_{p}(L, K, u) \\
& \quad \geq \int_{S^{n-1}} \frac{h_{L}(u)}{h_{K}(u) C_{p}(L)} \ln \left(\frac{h_{L}(u)}{h_{K}(u) C_{p}(L)}\right) d C_{p}(L, K, u),
\end{aligned}
$$


which is equivalent to the following inequality:

$$
\int_{S^{n-1}} \ln \left(\frac{h_{L}(u)}{h_{K}(u)}\right) d C_{p}^{*}(L, K, u) \geq \ln \left(\frac{C_{p}(L, K)}{C_{p}(L)}\right)
$$

with equality if and only if $K$ is homothetic to $L$. This completes the proof of the theorem.

Moreover, we show the logarithmic Minkowski type inequality for $p$-capacity.

Proof of Theorem 1.3 Let $q \in \mathbb{R}$ and $1<p<n$, consider the function

$$
G(q)=\int_{S^{n-1}}\left(\frac{h_{K}(u)}{h_{L}(u)}\right)^{q} \ln \left(\frac{h_{K}(u)}{h_{L}(u)}\right) d C_{p}(L, u)
$$

since $L \subseteq K$, the function $G(q)$ is nonnegative. If $\ln \left(\frac{h_{K}(u)}{h_{L}(u)}\right) \equiv 0, u \in \operatorname{supp} C_{p}(L, \cdot)$, then $G \equiv 0$, which implies that $G(1) \geq G(0)>0$. If $G(1)=G(0)$, then $K=L$. So we assume $G(1)>G(0)$.

We now claim that $G(q)$ is a log-convex function. In fact, let $t \in(0,1)$, by Hölder's inequality, we get that

$$
\begin{aligned}
G\left((1-t) q_{1}+t q_{2}\right)= & \int_{S^{n-1}}\left(\frac{h_{K}(u)}{h_{L}(u)}\right)^{(1-t) q_{1}+t q_{2}} \ln \left(\frac{h_{K}(u)}{h_{L}(u)}\right) d C_{p}(L, u) \\
\leq & \left(\int_{S^{n-1}}\left(\frac{h_{K}(u)}{h_{L}(u)}\right)^{q_{1}} \ln \left(\frac{h_{K}(u)}{h_{L}(u)}\right) d C_{p}(L, u)\right)^{1-t} \\
& \times\left(\int_{S^{n-1}}\left(\frac{h_{K}(u)}{h_{L}(u)}\right)^{q_{2}} \ln \left(\frac{h_{K}(u)}{h_{L}(u)}\right) d C_{p}(L, u)\right)^{t} \\
= & G\left(q_{1}\right)^{1-t} G\left(q_{2}\right)^{t} .
\end{aligned}
$$

Applying the Hadamard type inequality in [14], we have

$$
\frac{G(1)-G(0)}{\ln G(1)-\ln G(0)} \geq \int_{0}^{1} \int_{S^{n-1}}\left(\frac{h_{K}(u)}{h_{L}(u)}\right)^{q} \ln \left(\frac{h_{K}(u)}{h_{L}(u)}\right) d C_{p}(L, u) d q
$$

From Fubini-Tonelli's theorem, we obtain

$$
\begin{aligned}
G(0) & \geq G(1) \exp \left(-\frac{G(1)-G(0)}{\int_{0}^{1} \int_{S^{n-1}}\left(\frac{h_{K}(u)}{h_{L}(u)}\right) q \ln \left(\frac{h_{K}(u)}{h_{L}(u)}\right) d C_{p}(L, u) d q}\right) \\
& =G(1) \exp \left(-\frac{G(1)-G(0)}{\int_{S^{n-1}}\left(\frac{h_{K}(u)}{h_{L}(u)}-1\right) d C_{p}(L, u)}\right) .
\end{aligned}
$$

Notice that

$$
\frac{G(1)-G(0)}{\int_{S^{n-1}}\left(\frac{h_{K}(u)}{h_{L}(u)}-1\right) d C_{p}(L, u)} \leq \ln \left(\frac{h_{K}}{h_{L}}\right)_{\max } .
$$


Thus, by Theorem 1.2, we have

$$
\begin{aligned}
& \int_{S^{n-1}} \ln \left(\frac{h_{K}(u)}{h_{L}(u)}\right) d C_{p}^{*}(L, u) \\
& \geq \exp \left(-\ln \left(\frac{h_{K}}{h_{L}}\right)_{\max }\right) \frac{C_{p}(L, K)}{C_{p}(L)} \int_{S^{n-1}} \ln \left(\frac{h_{K}(u)}{h_{L}(u)}\right) d C_{p}^{*}(L, K, u) \\
& \geq \frac{\left(\frac{h_{K}}{h_{L}}\right)_{\text {average }}}{\left(\frac{h_{K}}{h_{L}}\right)_{\max }} \int_{S^{n-1}} \ln \left(\frac{h_{K}(u)}{h_{L}(u)}\right) d C_{p}^{*}(L, K, u) \\
& \geq \frac{\left(\frac{h_{K}}{h_{L}}\right)_{\text {average }}}{\left(\frac{h_{K}}{h_{L}}\right)_{\max }} \frac{1}{n-p} \ln \left(\frac{C_{p}(K)}{C_{p}(L)}\right) .
\end{aligned}
$$

Suppose $G(q) \equiv 0$, then $h_{K}(u)=h_{L}(u)$ for almost all $u$ in $L_{0} p$-capacitary measure of $L$, or equivalently with respect to the $p$-capacitary measure of $L$. This implies that $C_{p}(L, K)=$ $C_{p}(L)$. According to the equality condition of Minkowski inequality for $p$-capacity and $L \subseteq K$, we obtain $K=L$.

Assume that $K, L \in \mathcal{K}_{o}^{n}$ are arbitrary and $L$ is not included in $K$, then there exists $0<t<1$ such that $t L \subseteq K$. By (4.4), we have

$$
\int_{S^{n-1}} \ln \left(\frac{h_{K}(u)}{t h_{L}(u)}\right) d C_{p}^{*}(t L, u) \geq \frac{\left(\frac{h_{K}}{t h_{L}}\right)_{\text {average }}}{\left(\frac{h_{K}}{t h_{L}}\right)_{\max }} \frac{1}{n-p} \ln \left(\frac{C_{p}(K)}{C_{p}(t L)}\right)
$$

which is equivalent to the following inequality:

$$
\begin{aligned}
& \int_{S^{n-1}} \ln \left(\frac{h_{K}(u)}{h_{L}(u)}\right) d C_{p}^{*}(L, u) \\
& \quad \geq \frac{\left(\frac{h_{K}}{h_{L}}\right)_{\text {average }}}{\left(\frac{h_{K}}{h_{L}}\right)_{\max }} \frac{1}{n-p} \ln \left(\frac{C_{p}(K)}{C_{p}(L)}\right)+\ln t\left(1-\frac{\left(\frac{h_{K}}{h_{L}}\right)_{\text {average }}}{\left(\frac{h_{K}}{h_{L}}\right)_{\max }}\right) .
\end{aligned}
$$

Taking $t=\min _{u \in \operatorname{supp}} C_{p}(K,) \frac{h_{K}(u)}{h_{L}(u)}$, we obtain the second inequality, with equality if and only if $K$ and $L$ are homothetic.

Obviously, Theorem 1.3 implies the following corollary.

Corollary 4.1 Let $K, L \in \mathcal{K}_{o}^{n}$ and $1<p<n$. If there exists a constant $t>0$ such that $t L \subseteq K$ with $h_{K}(u)=h_{t L}(u)$ for $u$ in $L_{0} p$-capacitary measure of $L$, then

$$
\int_{S^{n-1}} \ln \left(\frac{h_{K}(u)}{h_{L}(u)}\right) d C_{p}^{*}(L, u) \geq \frac{1}{n-p} \ln \left(\frac{C_{p}(K)}{C_{p}(L)}\right) .
$$

Equality holds if and only if $K=t L$.

Finally, we shall give an application of Theorem 1.2. Let $\mathcal{F}_{n}$ be the set of all convex bodies with positive continuous curvature functions and $L$ be a convex body in $\mathcal{F}_{n}$ with a curvature function $f_{L}$. To simplify the notation, we write $K^{*}=\left\{x \in \mathbb{R}^{n}: x \cdot y \leq 1, y \in K\right\}$ to be the polar body of $K$ and $\Phi_{p}(L)=\int_{S^{n-1}} f_{L}^{\frac{n}{n+1}}\left|\nabla u_{L}\right|^{\frac{n p}{n+1}} d S(u)$. 
Theorem 4.2 If $K \in \mathcal{K}_{o}^{n}, 1<p<n$, and $L \in \mathcal{K}_{o}^{n} \cap \mathcal{F}_{n}$, then

$$
V(K) V\left(K^{*}\right) \geq \frac{1}{n(n-p)^{n}} \frac{\Phi_{p}(L)^{n+1}}{C_{p}(L)^{n}} \frac{V(K)}{c(p, K, L)}
$$

where $c(p, K, L):=\left(\exp \left(\int_{S^{n-1}} \ln \left(\frac{h_{K}(u)}{h_{L}(u)}\right)\right) d C_{p}^{*}(L, K, u)\right)^{n}$.

Proof Let $K$ and $L$ be distinct, by Theorem 1.2, we have

$$
\begin{aligned}
\exp \left(\int_{S^{n-1}} \ln \left(\frac{h_{K}(u)}{h_{L}(u)}\right)\right) d C_{p}^{*}(L, K, u) & \geq \frac{C_{p}(L, K)}{C_{p}(L)} \\
& =\frac{1}{(n-p) C_{p}(L)} \int_{S^{n-1}} h_{K}(u) f_{L}\left|\nabla u_{L}\right|^{p} d S(u) .
\end{aligned}
$$

Using the reverse Hölder inequality, we obtain

$$
\begin{aligned}
\int_{S^{n-1}} h_{K} f_{L}\left|\nabla u_{L}\right|^{p} d S(u) & \geq\left(\int_{S^{n-1}} h_{K}^{-n} d S(u)\right)^{-\frac{1}{n}}\left(\int_{S^{n-1}} f_{L}^{\frac{n}{n+1}}\left|\nabla u_{L}\right|^{\frac{n p}{n+1}} d S(u)\right)^{\frac{n+1}{n}} \\
& =\left(n V\left(K^{*}\right)\right)^{-\frac{1}{n}} \Phi_{p}(L)^{\frac{n+1}{n}} .
\end{aligned}
$$

Thus

$$
\exp \left(\int_{S^{n-1}} \ln \left(\frac{h_{K}(u)}{h_{L}(u)}\right)\right) d C_{p}^{*}(L, K, u) \geq \frac{1}{(n-p) C_{p}(L)}\left(n V\left(K^{*}\right)\right)^{-\frac{1}{n}} \Phi_{p}(L)^{\frac{n+1}{n}}
$$

which implies that

$$
\left(\exp \left(\int_{S^{n-1}} \ln \left(\frac{h_{K}(u)}{h_{L}(u)}\right)\right) d C_{p}^{*}(L, K, u)\right)^{n} \geq \frac{1}{n(n-p)^{n} C_{p}(L)^{n} V\left(K^{*}\right)} \Phi_{p}(L)^{n+1}
$$

This gives the desired inequality.

For the case $K=L \in \mathcal{K}_{o}^{n} \cap \mathcal{F}_{n}$, according to the above proof, we also obtain the desired inequality.

Taking $K=L$, we obtain the following result.

Corollary 4.2 If $K \in \mathcal{K}_{o}^{n} \cap \mathcal{F}_{n}$ and $1<p<n$, then

$$
V(K) V\left(K^{*}\right) \geq \frac{1}{n(n-p)^{n}} \frac{V(K) \Phi_{p}(K)^{n+1}}{C_{p}(K)^{n}} .
$$

\section{Acknowledgements}

We would like to thank the referees for valuable suggestions and comments that led to the improvement of the original manuscript.

\section{Funding}

The Natural Science Foundation of China (Grant No. 71762001), the Science and Technology Project of Education Department of Jiangxi Province (Grant No. GJJ180414), and the PhD research startup foundation of East China University of Technology (Grant No. DHBK2018050). 
Availability of data and materials

Not applicable.

\section{Competing interests}

The author declares to have no competing interests.

\section{Authors' contributions}

The author was the only one to contribute in writing this article. The author read and approved the final manuscript.

\section{Publisher's Note}

Springer Nature remains neutral with regard to jurisdictional claims in published maps and institutional affiliations.

Received: 4 July 2019 Accepted: 12 February 2020 Published online: 25 February 2020

\section{References}

1. Akman, M., Gong, J., Hineman, J., Lewis, J., Vogel, A.: The Brunn-Minkowski inequality and a Minkowski problem for nonlinear capacity. In press. arXiv:1709.00447

2. Böröczky, J., Hegedűs, P., Zhu, G.: The discrete logarithmic Minkowski problem. Int. Math. Res. Not. 6, 1807-1838 (2014)

3. Böröczky, J., Lutwak, E., Yang, D., Zhang, G.: The log-Brunn-Minkowski inequality. Adv. Math. 231, 1974-1997 (2012)

4. Böröczky, J., Lutwak, E., Yang, D., Zhang, G.: The logarithmic Minkowski problem. J. Am. Math. Soc. 26, 831-852 (2013)

5. Caffarelli, L.: Interior $W_{2}, p$-estimates for solutions of the Monge-Ampère equation. Ann. Math. 131, 135-150 (1990)

6. Caffarelli, L., Jerison, D., Lieb, E.: On the case of equality in the Brunn-Minkowski inequality for capacity. Adv. Math. 117, 193-207 (1996)

7. Colesanti, A.: Brunn-Minkowski inequalities for variational functionals and related problems. Adv. Math. 194, 105-140 (2005)

8. Colesanti, A., Cuoghi, P.: The Brunn-Minkowski inequality for the $n$-dimensional logarithmic capacity of convex bodies. Potential Anal. 22, 289-304 (2005)

9. Colesanti, A., Nyström, K., Salani, P., Xiao, J., Yang, D., Zhang, G.: The Hadamard variational formula and the Minkowski problem for $p$-capacity. Adv. Math. 285, 1511-1588 (2015)

10. Colesanti, A., Salani, P.: The Brunn-Minkowski inequality for p-capacity of convex bodies. Math. Ann. 327, 459-479 (2003)

11. Cover, T., Thomas, J.: Elements of Information Theory, 2nd edn. Wiley-Interscience, Hoboken (2006)

12. Dahlberg, B.: Estimates of harmonic measure. Arch. Ration. Mech. Anal. 65, 275-288 (1977)

13. Evans, L., Gariepy, R.: Measure Theory and Fine Properties of Functions. CRC Press, Boca Raton (1992)

14. Gill, P., Pearce, C., Pečarić, J.: Hadamard's inequality for r-convex functions. J. Math. Anal. Appl. 215, 461-470 (1997)

15. Han, H., Ye, D., Zhang, N.: The p-capacitary Orlicz-Hadamard variational formula and Orlicz-Minkowski problems. Calc. Var. Partial Differ. Equ. 57, 1-31 (2018)

16. Hong, H., Ye, D.: Sharp geometric inequalities for the general p-affine capacity. J. Geom. Anal. 28, 2254-2287 (2018)

17. Jerison, D.: A Minkowski problem for electrostatic capacity. Acta Math. 176, 1-47 (1996)

18. John, F.: Polar correspondence with respect to a convex region. Duke Math. J. 3, 355-369 (1937)

19. Luo, X., Ye, D., Zhu, B.: On the polar Orlicz-Minkowski problems and the $p$-capacitary Orlicz-Petty bodies. Indiana Univ. Math. J. In press

20. Lutwak, E., Yang, D., Zhang, G.: Volume inequalities for subspaces of Ip. J. Differ. Geom. 68, 159-184 (2004)

21. Saroglou, C.: Remarks on the conjectured log-Brunn-Minkowski inequality. Geom. Dedic. 177, 353-365 (2015)

22. Schneider, R.: Convex Bodies: The Brunn-Minkowski Theory, 2nd edn. Cambridge University Press, New York (2014)

23. Stancu, A.: The logarithmic Minkowski inequality for non-symmetric convex bodies. Adv. Appl. Math. 73, 43-58 (2016)

24. Thompson, A.C.: Minkowski Geometry, Encyclopedia of Mathematics and Its Applications. Cambridge University Press, Cambridge (1996)

25. Wang, X., Xu, W., Zou, J.: Some logarithmic Minkowski inequalities for nonsymmetric convex bodies. Sci. China Math. 60, 129-144 (2017)

26. Xi, D., Leng, G.: Dar's conjecture and the log-Brunn-Minkowski inequality. J. Differ. Geom. 103, 145-189 (2016)

27. Xiao, J.: p-capacity vs surface-area. Adv. Math. 308, 1318-1336 (2017)

28. Xiong, G.: Extremum problems for the cone volume functional for convex polytopes. Adv. Math. 225, 3214-3228 (2010)

29. Xiong, G., Xiong, J., Xu, L.: The Ip capacitary Minkowski problem for polytopes. J. Funct. Anal. 277, 3131-3155 (2019)

30. Zhu, G.: The logarithmic Minkowski problem for polytopes. Adv. Math. 262, 909-931 (2014)

31. Zou, D., Xiong, G.: The Ip Minkowski problem for the electrostatic $p$-capacity. J. Differ. Geom. (2019) In press 